\section{Impacto del control de tabaco en los costos asistenciales del infarto agudo de miocardio en Uruguay: costos en base a Grupos Relacionados por el Diagnóstico}

\author{
Impact of tobacco control on healthcare costs for \\ acute myocardial infarction in Uruguay: costs \\ based on Diagnosis-Related Groups
}

Impacto do controle do tabaco sobre os custos da atenção ao infarto agudo do miocárdio no Uruguai: custos com base em Grupos Relacionados pelo Diagnóstico
Elbio Paolillo-Cabrera 1

Frank Torres 1,2

Federico Machado 1,2

Alberto Scasso 1

Alicia Alvarado 1

Diego Genta 1

Pablo Tort 1

Silvina Tortorella 1

Sandra Laluz 1

Daniela Cabrera 1

Edgardo Sandoya 2

doi: 10.1590/0102-311X00149019

\title{
Resumen
}

El tabaco es la principal causa modificable de enfermedad cardiovascular, cáncer y enfermedades respiratorias, por lo que es un serio problema de salud pública universal. En 2006, Uruguay implementó el Convenio Marco para el Control del Tabaco de la Organización Mundial de la Salud (CMCT-OMS), $y$ consiguió un descenso de la tasa de fumadores y mejoras en la salud cardiovascular y respiratoria. Se investigó el impacto clínico y económico de las medidas de control de tabaco sobre los costos asistenciales del infarto agudo de miocardio, que se redujo un 17\%. No se incluyeron los costos evitados por otras patologías. Se investigó lo ocurrido en una institución sanitaria y se proyectó a toda la población del país. El análisis de costos se realizó usando la metodología del sistema de los Grupos Relacionados por el Diagnóstico (GRD), combinada con los informes contables de la institución. Además de los costos de internación, se incluyeron los relacionados con los traslados, los de los procedimientos cardiovasculares invasivos y los gastos sanitarios de los 12 meses posteriores al evento agudo. El costo por paciente fue de USD 12.037. Considerando un descenso de 500 infartos por año, el ahorro anual estimado es de USD 6.000.000 en costos asistenciales de los infartos evitados; a lo que debería agregarse otros ahorros de costos por pérdida laboral, discapacidad posterior $y$ años de vida prematura perdidos por fallecimiento del paciente. La exitosa política de control del tabaco ha sido la principal medida de salud pública en los últimos 30 años en nuestro país. Esta investigación busca contribuir al camino trazado por la Organización Mundial de la Salud (OMS).

Tabaco; Costos y Análisis de Costo; Infarto Agudo de Miocardio

\author{
Correspondencia \\ E. Paolillo-Cabrera \\ Asistencial Médica Departamental de Maldonado. \\ Camino a la Laguna, Parada 36, Punta del Este / Maldonado - \\ 20005, Uruguay. \\ elbio.paolillo@gmail.com \\ 1 Asistencial Médica Departamental de Maldonado, Punta del \\ Este, Uruguay. \\ 2 Centro Latinoamericano de Economía Humana, Punta del \\ Este, Uruguay.
}




\section{Introducción}

El tabaco es la principal causa modificable de enfermedad cardiovascular, cáncer y enfermedades respiratorias, por lo que su consumo constituye un serio problema de salud pública a escala universal y su resolución afecta múltiples intereses $1,2,3$.

El tabaquismo es responsable del $80 \%$ de las muertes por enfermedad pulmonar obstructiva crónica, $40 \%$ de las ocurridas por enfermedad cardiovascular precoz y $30 \%$ de las debidas a cáncer. Además, el tabaco aumenta la mortalidad en los recién nacidos de madres fumadoras, y la de las personas expuestas al humo de segunda mano 4.

En Uruguay se ha estimado que ocurren entre 4.800 y 6.500 muertes atribuibles al consumo de tabaco cada año, de las cuales un $35 \%$ obedecen a cáncer, $29 \%$ a enfermedad respiratoria y $28 \%$ a enfermedad cardiovascular 2,3. El tabaco es también el principal determinante de años de vida perdidos, y de años de vida saludable perdidos en el país, por su impacto a nivel cardio y cerebrovascular 5 .

Además de los daños a la salud, el tabaco determina la pérdida de productividad, tanto del fumador, como de los individuos expuestos al humo de segunda mano. De esta manera, al peso económico que impone el consumo de tabaco a la sociedad, debido a los gastos médicos de la enfermedad y sus complicaciones, se deben agregar los que se derivan de la pérdida de productividad y los años potenciales de vida perdidos por incapacidad o muerte prematura.

Los costos de la atención médica atribuibles al consumo de tabaco en países desarrollados representan hasta un 1,1\% del Producto Interno Bruto (PIB) 2. Las políticas para el control del consumo de tabaco, implementadas en Uruguay en el período reciente, toman como piedra angular el Convenio Marco para el Control del Tabaco de la Organización Mundial de la Salud (CMCT-OMS), adoptado en el año 2003, y puesto en vigor en el año 2005 6. En el año 2006 Uruguay comenzó la implementación de un amplio programa de control del tabaco, que ha tenido resultados beneficiosos en diversos aspectos. Dado que no se ha explorado el impacto que esto ha tenido desde el punto de vista económico, en la presente investigación nos propusimos estimarlo desde la óptica del sistema sanitario del país.

\section{Características de Uruguay}

Uruguay es un país de unos 3.400 .000 habitantes, con un $52 \%$ de su población de sexo femenino ${ }^{7}$. En la capital, Montevideo, reside el $40 \%$ de la población y es allí donde se concentra el $85 \%$ de los Institutos de Medicina Altamente Especializada (IMAE), los que realizan los procedimientos cardiovasculares invasivos. Maldonado es uno de los 19 departamentos del país, y se sitúa a $135 \mathrm{~km}$ de Montevideo, teniendo una población de 165.000 habitantes, que en verano llega a 400.000 individuos, dada la presencia de importantes zonas turísticas, entre la que destaca la ciudad de Punta del Este.

La atención de salud del país se brinda a través del Sistema Nacional Integrado de Salud (SNIS) desde el año 2008 8, que consta de un subsector privado que da cobertura a 65\% de la población, y uno público que da cobertura al 35\% restante. El Fondo Nacional de Recursos (FNR) es un reaseguro de financiación mixta, y gestión estatal, que financia los procedimientos cardiovasculares invasivos a toda la población del país.

Como resultado de las políticas de control de tabaco 9, desde 2006 se ha observado descenso de la tasa de fumadores adultos, que pasó de un 35\% en 2005 a un 22\% en 2014 10. Asimismo, se ha comprobado una elevada tasa de acatamiento de la prohibición de fumar en espacios cerrados de uso público, que fue superior al $96 \%$ y, como consecuencia de ello, una marcada reducción en la contaminación del aire con partículas en suspensión respirables (material particulado $2,5-\mathrm{MP}_{2,5}$ ), que pasó de $210 \mu \mathrm{g} / \mathrm{m}^{3}$ antes de la prohibición, a $18 \mu \mathrm{g} / \mathrm{m}^{3}$ después de ella 11 . A nivel sanitario se observó un efecto favorable en la salud respiratoria, con una reducción de $15 \%$ en las consultas pre-hospitalarias por broncoespasmo, así como una reducción en las dosis de fármacos administrados para su manejo ${ }^{12}$. En el aspecto de la salud cardiovascular se observó una reducción de 17\% en los ingresos por infarto agudo de miocardio (IAM), beneficio que comenzó a operar inmediatamente después de la introducción de las medidas, y se mantenía en los 48 meses siguientes 13 .

Los cardiólogos que integran este grupo tienen una extensa experiencia clínica y han investigado y publicado artículos sobre los estilos de vida y la patología cardiovascular en la zona y en todo el Uruguay 14,15 . 


\section{Objetivo}

El objetivo de esta investigación es estimar los costos asistenciales de las medidas de control de tabaco en la reducción de los IAM.

\section{Material y método}

Se utilizó la metodología de costos de los Grupos Relacionados por el Diagnóstico (GRD).

\section{Definición de los GRD}

Los GRD fueron creados por R. Fetter en 1980. La definición original es: "Los GRD son un sistema de clasificación de pacientes, que se basa en la agrupación de los mismos, que egresan de un hospital, a partir de la información contenida en la hoja de cierre de la historia clínica Conjunto Mínimo de Datos Básicos (CMDB), conformando grupos homogéneos, en cuanto a consumo de recursos y con coherencia clínica" 16 (p. 3).

En investigaciones previas, algunos de los autores del presente estudio han aplicado dicha metodología para la mejora en la gestión, sistemas de información y costos en distintas patologías 17,18.

Esta herramienta de gestión clínica y de costos ha tenido poca difusión hasta el momento en América del Sur, salvo escasas excepciones 19,20,21.

Se realizó una estimación de los costos sanitarios, desde la perspectiva del SNIS, a partir del análisis de lo ocurrido en los asociados de una institución sanitaria: la Asistencial Médica Departamental de Maldonado (AMDM). Una Institución de Asistencia Médica Colectiva (IAMC), integrante del subsector privado del SNIS, que brinda cobertura sanitaria a 80.000 asociados, lo que luego se proyectó a la población de todo el país.

\section{Muestra empleada}

El estudio se realizó sobre el total de pacientes ingresados por IAM a la AMDM, durante un período de 5 años, de los cuales se determinó los costos del evento agudo, a lo que se añadieron los costos estimados en el año siguiente. Para ello, se generó un listado de todos los pacientes internados entre el 01/Ene/2011 y el 31/Dic/2015, cuyo diagnóstico fuese IAM. A partir del listado, se revisaron todas las historias clínicas en papel, lo que se complementó con el análisis de la historia clínica electrónica en caso de ser necesario.

\section{- Criterios de inclusión y exclusión}

Se incluyeron en el estudio los casos que al egreso tuviesen IAM como diagnóstico de ingreso, y que cumpliesen con los criterios de la definición universal de IAM 22. Se excluyeron los pacientes que ingresaron por otra razón y desarrollaron el IAM como complicación durante la internación. Se adoptó este criterio, dado que el mismo fue el empleado en la investigación previa, que evaluó el impacto en los ingresos por IAM en todo el país.

\section{Variables analizadas}

Con la finalidad de comparar las características de la muestra con las del total de ingresos por IAM en el país, en cada caso se analizó edad, sexo, tipo de IAM (con y sin elevación de ST), clase funcional Killip y Kimball (KK) y procedimientos cardiovasculares invasivos: cineangiocoronariografía (CACG), angioplastia coronaria (ATC), cirugía de revascularización miocárdica (CRM). A los efectos de su clasificación por GRD, se analizaron días de internación, área de internación (convencional e intensiva) y situación al alta (vivo o fallecido). A los efectos de complementar el análisis de los costos, se analizaron los traslados realizados, reingresos vinculados a la misma patología, consultas ambulatorias, medicación recibida y estudios realizados en los 12 meses siguientes al evento, además de la situación al año (vivo o fallecido). 


\section{- Definición de variables}

Se consideró como IAM con elevación del segmento ST, cuando se comprobó ese hallazgo en el electrocardiograma (ECG), y como IAM sin elevación de ST, cuando no lo presentaba. Se consideró como traslados vinculados al evento agudo, aquellos efectuados para la realización de procedimientos invasivos, tanto hacia el IMAE, como de regreso del mismo. La clase funcional se consideró de acuerdo a la clasificación de Killip y Kimball en el momento del ingreso.

\section{- Recolección de variables}

Las variables referidas a la internación y las de los traslados se recogieron de la historia clínica en papel y de la historia clínica electrónica, al igual que la situación del paciente al año. Cuando este último dato no estuvo disponible, se verificó si había fallecido en la base de datos de la Comisión para la Salud Cardiovascular, que registra en su base de datos todos los fallecimientos de causa cardiovascular ocurridos en el país. Las variables relacionadas a los procedimientos invasivos se relevaron de la historia clínica y a partir de los datos proporcionados por el FNR. Las consultas ambulatorias y los estudios realizados en el año siguiente a la internación se estimaron a partir de datos de uso de procedimientos. Los costos de la medicación ambulatoria en el año siguiente al IAM se estimaron a partir de la medicación que debe recibir un paciente en prevención secundaria, de acuerdo a lo establecido en las guías de práctica clínica 23 .

\section{Metodología de costos utilizada}

El cálculo del costo asistencial de cada paciente incluyó cuatro componentes, comprendiendo los costos de: (1) Internación aguda: los que se calcularon mediante el sistema de los GRD y los costos institucionales. A los costos de la internación inicial, se añadieron los de eventuales internaciones, relacionadas con el mismo evento, por ejemplo, cuando tras la realización de un procedimiento invasivo en un IMAE, el paciente volvió para completar la evolución aguda; (2) Traslados entre instituciones; (3) Procedimientos cardiovasculares invasivos; y (4) Manejo en el año posterior al ingreso. En este caso, a los gastos derivados del manejo ambulatorio se añadieron los ingresos de naturaleza cardiovascular producidos en ese período.

A partir de los resultados observados, se realizó la extrapolación de los costos al total de ingresos por IAM evitados en el país, dato disponible a partir de la investigación previa, que evaluó el impacto de la medida en los ingresos por IAM 13.

\section{Definición de costos}

Los costos de internación se establecieron usando el sistema de clasificación de pacientes por GRD para clasificar los egresos hospitalarios y una planilla de costos, basada en el balance de cada institución, denominada Estructura de Costos de Atención a Socios (ECAS). Esta planilla considerada extracontable es auditada anualmente por la autoridad sanitaria del SNIS. El sistema de costos por GRD aplicado, combina el sistema Top-down Costing y Activity-bases Costing con la información clínica de los pacientes asistidos y esto posibilita tener el costo promedio por grupo de pacientes, en este caso los del IAM. Se adjunta en la Figura 1 la publicación de la Universidad Kebangssan de Malaysia 24,25,26.

\section{- Internación aguda}

\section{a) Descripción del método de costos por GRD}

La metodología de costos por GRD para pacientes internados en un hospital tiene dos componentes fundamentales que son: el Conjunto Mínimo de Datos Básicos (CMDB) y la planilla ECAS.

El CMDB es una planilla estructurada, que recopila variables administrativas y variables clínicas del paciente y de su proceso de internación en un hospital. Dicho CMDB es indispensable para obtener el GRD de un egreso hospitalario; consiste en variables como la edad, sexo, tipo de ingreso, 


\section{Figura 1}

Flujo en el sistema Casemix.

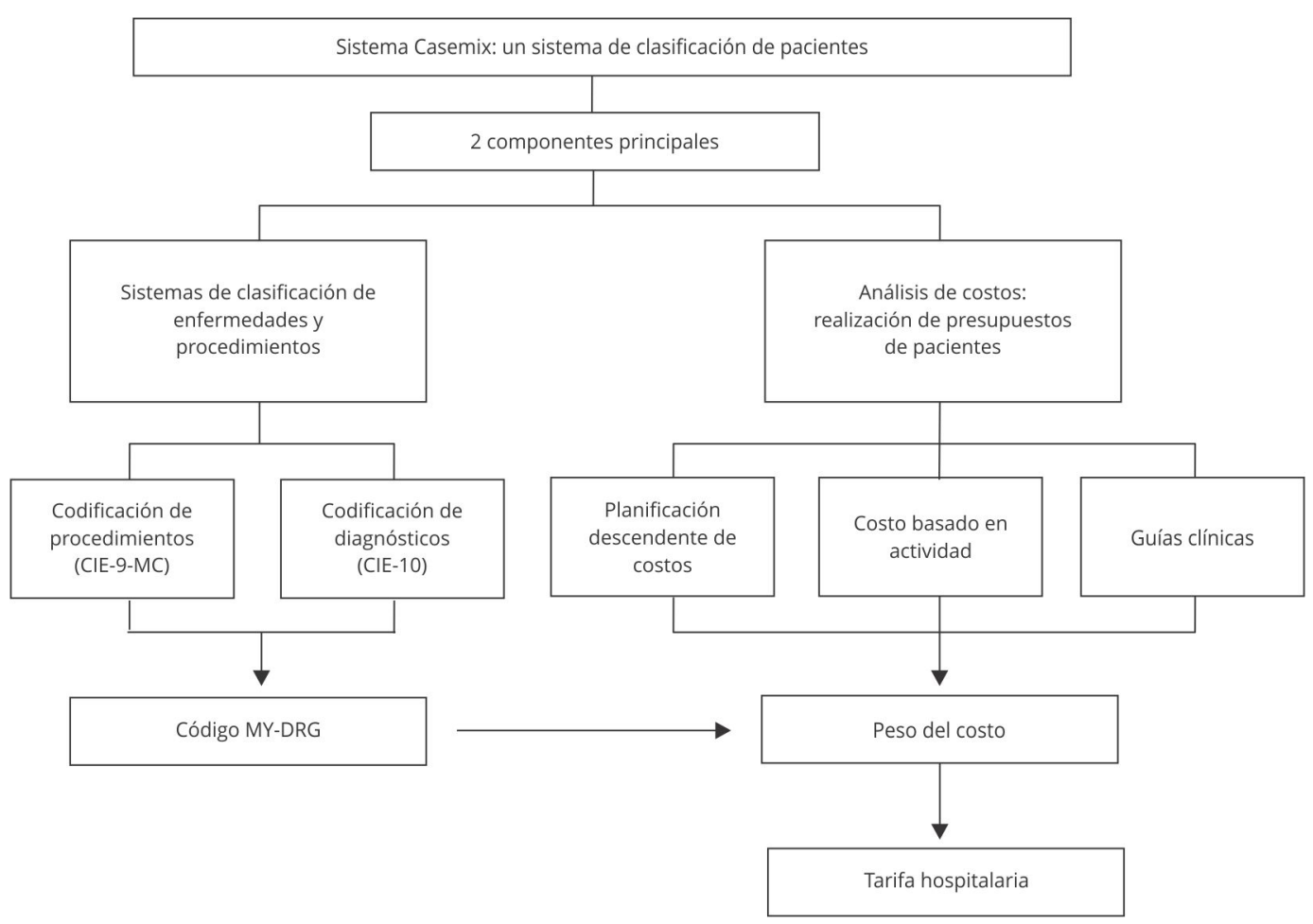

Nota: Casemix es un sistema de clasificación de pacientes, en el que existen dos componentes principales, a saber: codificación clínica y análisis de costos. El proceso de codificación se efectúa de acuerdo a la Clasificación Internacional de Enfermedades - 10a revisión (CIE-10) 27 para la codificación de diagnósticos, y la Modificación Clínica de la Clasificación Internacional de Enfermedades - 9a revisión (CIE-9-MC) 28 para la codificación de los procedimientos. Por otra parte, los métodos de costo que son usualmente empleados en el Sistema Casemix son: planificación descendente de costos (Top-down Costing) y costos basados en la actividad (Activity-based Costing). En el Sistema Casemix, la información del proceso de codificación clínica es usada para generar el código MY-DRG, y estos códigos serán asignados a una tarifa hospitalaria, según el peso del costo calculado en el apartado del análisis de costo.

tipo de egreso, duración de la estadía, diagnósticos codificados con la Clasificación Internacional de Enfermedades - 10a revisión (CIE-10) 27, y procedimientos codificados con la Modificación Clínica de la Clasificación Internacional de Enfermedades - 9ạ revisión (CIE-9-MC) 28, etc.

La planilla ECAS surge como exigencia del Ministerio de Salud Pública del Uruguay a las Instituciones de Asistencia Médica Colectiva (IAMC) que integran el SNIS. La referida planilla se elabora en base al balance económico financiero de cada institución y agrupa los rubros contables en distintos centros de costos generales, intermedios y finales. Las instituciones prestadoras, que a su vez son aseguradoras, deben reportar esta planilla anualmente a la Dirección General de Salud.

A continuación, describiremos paso a paso cómo distribuir el costo total de cada centro de costos finales entre los pacientes internados, con el fin de obtener el costo individual de cada egreso de internación. Para ello, debemos trabajar con el CMDB que contiene todos los egresos de un año y la planilla ECAS que corresponde al mismo período.

En la planilla del CMDB agregamos cuatro columnas a las que nombramos como: "Días cuidados moderados"; "Días UCI Adultos”; "Días UCI Pediátrica”; "Quirúrgico”. 
Asignamos los "Días cuidados moderados", restando a los días de internación total del paciente, los días de unidad de cuidados intensivos (UCI) adultos y los días de UCI pediátrica.

El CMDB contiene como variable los días de UCI, tanto de adultos, como de pediatría; de ahí tomamos el valor para completar las columnas "Días UCI Adultos" y "Días UCI Pediátrica".

Se ordena toda la planilla, según la variable tipo de paciente Médico/Quirúrgico (esto lo define el sistema GRD); ello determina el valor que va a tomar la columna "Quirúrgico". Si el paciente es Médico asignamos 0 (cero); si el paciente es Quirúrgico asignamos 1.

El siguiente paso es calcular a partir de los centros de costos finales de ECAS, los costos unitarios por día de internación para Cuidados Moderados, UCI Adultos y UCI Pediátrica; y el costo del paciente Quirúrgico.

- Tomamos el costo total del centro de costos Cuidados Moderados y lo dividimos por el total de días de Cuidados Moderados, tomados de la columna "Días Cuidados Moderados", para obtener el costo/ día en Cuidados moderados.

- El costo total del centro de costos de UCI Adultos dividido el total de días de la columna "Días UCI Adultos", para obtener el costo/día en UCI Adultos.

- El costo total del centro de costos de UCI Pediátrica, dividido el total de días de la columna "Días UCI Pediátrica", para obtener el costo/día en UCI Pediátrica.

- El costo total del centro de costos de Block Quirúrgico lo repartimos proporcionalmente entre las cirugías ambulatorias y las cirugías con internación. El costo total de las cirugías con internación lo dividimos por el número total de egresos quirúrgicos del CMDB, para obtener el costo unitario por egreso Quirúrgico.

Obtenidos los 4 costos unitarios, agregamos a la planilla del CMDB cuatro columnas nombradas de la siguiente manera:

- "USD Cuidados Moderados";

- "USD UCI Adultos";

- "USD UCI Pediátrica";

- "USD Quirúrgico".

Seguido a esto, vamos a calcular el costo de cada paciente en cada uno de los cuatro rubros mencionados; para luego sumarlos y obtener el costo total del paciente en cada una de las internaciones en el hospital.

1. Se multiplica el costo/día de Cuidados Moderados, por el valor de la columna "Días Cuidados Moderados" y se obtiene el "USD Cuidados Moderados" para cada paciente de la planilla.

2. Se multiplica el costo/día de UCI Adultos, por el valor de la columna "Días UCI Adultos" y se obtiene el "USD UCI Adultos" para cada paciente de la planilla.

3. Se multiplica el costo/día de UCI Pediátrica, por el valor de la columna "Días UCI Pediátrica” y se obtiene el "USD UCI Pediátrica" para cada paciente de la planilla.

4. Se asigna el costo unitario de Block Quirúrgico para pacientes internados, a cada paciente identificado como quirúrgico, y se logra el "USD Quirúrgico" de cada paciente.

Finalmente, se suman los costos de cada paciente: "USD Cuidados Moderados" + "USD UCI Adultos" o "USD UCI Pediátrica" + "USD Quirúrgico".

De este modo, se compone el costo total de cada egreso.

Se adjunta Figura 2 que describe el método de costos, usando GRD y ECAS.

\section{- Traslados}

A ello, se adicionó el costo del traslado en unidad especializada realizado al IMAE donde se efectuó el procedimiento cardiovascular invasivo. Dicho costo fue proporcionado por el servicio de traslado de AMDM. El traslado "especializado" en nuestro medio se entiende como aquel realizado en una ambulancia equipada con todos los elementos para atender al paciente crítico, con enfermero y con médico especialista en pacientes cardiológicos complejos. También, se consideran traslados de menor complejidad los que se realizan en las mismas unidades, pero involucran a pacientes estables, los que se realizan con personal de enfermería, pero no incluyen médico especialista. 


\section{Figure 2}

Obtención de los costos unitarios usando Grupos Relacionados por el Diagnóstico (GRD) y Estructura de Costos de Atención a Socios (ECAS).

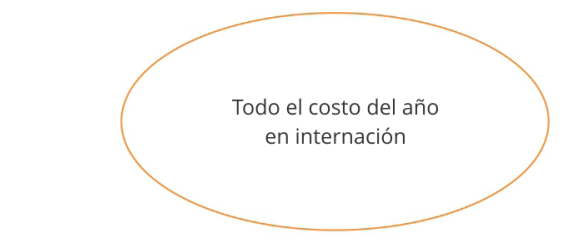

\section{ECAS}

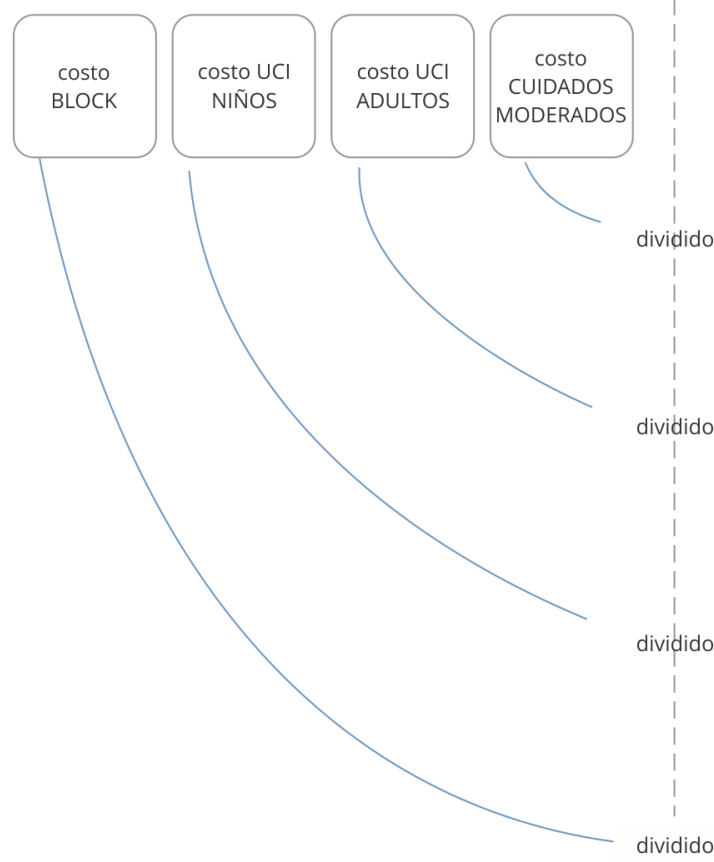

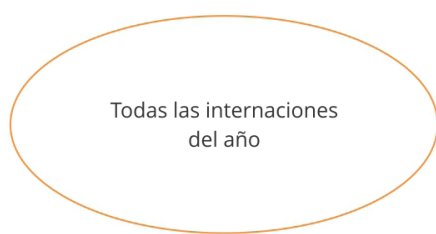

Días de internación totales (DCO)
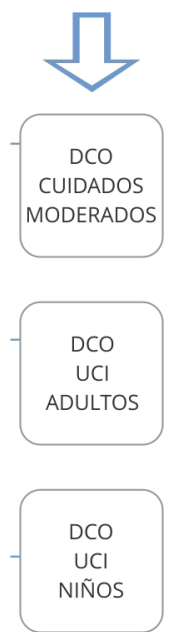

\section{GRD}

Médicos

Quirúrgicos

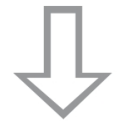

Egresos Quirúrgicos

UCl: unidad de cuidados intensivos.

\section{- Procedimientos cardiovasculares invasivos}

Los costos de procedimientos de este tipo realizados a los pacientes de la muestra fueron proporcionados por el FNR.

\section{- Manejo en el año posterior}

Cuando los pacientes tuvieron un nuevo ingreso cardiovascular el costo del mismo se realizó aplicando la metodología de GRD, de igual manera que en el ingreso inicial. A ello se agregaron los costos estimados, referidos a consultas ambulatorias, medicación y estudios complementarios realizados.

El costo final de cada paciente resultó de la suma de los cuatro tipos de costos referidos antes, y se expresan en dólares norteamericanos (USD), con el fin de facilitar la comparación. 


\section{- Extrapolación de costos al total del país}

La misma se realizó proyectando los costos de un paciente individual al total de ingresos estimados por IAM que se evitan cada año en el país.

Se comparan las características de los pacientes de la muestra y las disponibles de los pacientes de la investigación que abarcó a los pacientes de todo el país, a los efectos de ver similitudes y diferencias entre ambas.

\section{Resultados}

\section{Características de la muestra}

Al cabo de los 5 años analizados hubo 427 pacientes con diagnóstico al egreso de IAM, de los cuales 14 tuvieron un IAM secundario, o no cumplieron con los criterios de IAM adoptados, por lo que finalmente fueron 413 los incluidos en el estudio. De ellos, 292 (71\%) fueron hombres y 121 (29\%) mujeres y su edad media fue de 64,7 años (DE \pm 13,2), con un rango de 31 a 97 años. En 52\% de los casos $(\mathrm{n}=213)$ se trató de un IAM con elevación de ST. La clase funcional fue KK I en $87 \%(\mathrm{n}=359)$, KK II en $9 \%(n=36)$, KK III en 3\% ( $n=11)$ y KK IV en 2\% ( $n=7)$. El 75\% ( $n=312)$ de los pacientes fueron trasladados, mientras que $69 \%$ del total $(\mathrm{n}=287)$ fueron sometidos a procedimientos cardiovasculares intervencionistas (CACG $\mathrm{n}=279$, ATC $\mathrm{n}=180$, CRM $\mathrm{n}=39$ ). La mortalidad durante la internación fue de $5 \%$ (19 casos), siendo mayor entre mujeres (9\%, 11 muertes) que entre hombres (3\%, 8 muertes), mientras que la mortalidad al año fue de $4 \%$.

En la Tabla 1 se presentan las características disponibles de los pacientes de AMDM y del total del país.

Tabla 1

Características de los pacientes con infarto agudo de miocardio en la Asistencial Médica Departamental de Maldonado (AMDM) y en Uruguay.

\begin{tabular}{|c|c|c|}
\hline Características & AMDM & Uruguay \\
\hline $\mathrm{n}$ & 413 & 7.949 \\
\hline Sexo femenino & $29 \%$ & $35 \%$ \\
\hline Promedio & 64,7 & 66,7 \\
\hline \multicolumn{3}{|l|}{ Edad (años) } \\
\hline Desvío estándar & 13,2 & 12,6 \\
\hline Rango & $31-97$ & $21-103$ \\
\hline \multicolumn{3}{|c|}{ Clase funcional de Killip y Kimball (se reporta solo a nivel privado) } \\
\hline I & $87 \%$ & $81 \%$ \\
\hline II & $9 \%$ & $12 \%$ \\
\hline III & $3 \%$ & $4 \%$ \\
\hline IV & $2 \%$ & $3 \%$ \\
\hline \multicolumn{3}{|l|}{ Tipo de infarto } \\
\hline Con elevación de ST & $52 \%$ & $70 \%$ \\
\hline Sin elevación de ST & $48 \%$ & $30 \%$ \\
\hline Procedimientos cardiovasculares intervencionistas & $68 \%$ & $66 \%$ \\
\hline Mortalidad hospitalaria & $5 \%$ & $6 \%$ \\
\hline
\end{tabular}




\section{Estimación de costos de los pacientes de AMDM}

De acuerdo a la distribución de los pacientes en GRD, un 78\% de ellos fue catalogado como IAM leve $(\mathrm{n}=321), 14 \%$ como IAM moderado $(\mathrm{n}=57)$ y $8 \%$ como IAM severo $(\mathrm{n}=35)$.

En la Tabla 2 se presentan los costos promedio del evento agudo por paciente que incluye internación, traslados y procedimientos cardiovasculares invasivos.

Se incluye además los costos promedio por paciente en el año siguiente al evento agudo en sus cuatro componentes, internaciones, traslados, consultas y fármacos.

El costo total promedio por paciente al año fue de USD 12.037.

\section{Estimación de costos extrapolada al país}

A partir de los datos de la investigación previa que analizó los ingresos por IAM antes y después de las medidas de marzo de 2006, se estima que luego de la misma hubo una reducción anual de unos 500 ingresos por IAM en el país 12. De acuerdo a estos hallazgos, cada año se ahorrarían aproximadamente USD 6.000.000 por los costos de atención de los IAM evitados en el país.

Tabla 2

Costo promedio, em dólares norteamericanos (USD), por paciente del evento agudo y seguimiento.

\begin{tabular}{lccc}
\hline & Evento agudo (USD) & Seguimiento (USD) & Total (USD) \\
\hline Internación & 3.361 & 1.445 & 4.806 \\
Traslados & 1.343 & 1.409 & 2.754 \\
Procedimientos & 3.901 & - & 3.901 \\
Consultas & - & 54 & 54 \\
Fármacos & - & 524 & 524 \\
Total & 8.605 & 3.432 & 12.037 \\
\hline
\end{tabular}

\section{Discusión}

Las medidas de control de tabaco han tenido impacto positivo en la salud de la población del país, tanto en la esfera respiratoria, como la cardiovascular, pero hasta ahora no se había podido determinar el correlato económico de dicho beneficio sanitario. En este estudio se investigó el impacto económico de las mismas en relación al IAM, que en investigaciones previas había mostrado una reducción de $17 \%$ en los ingresos. De acuerdo a lo estimado, cada año se ahorrarían unos USD 6.000.000 al SNIS. Dado que han transcurrido 12 años desde la implementación de las medidas, el país habría ahorrado por lo menos USD 72.000.000 en costos asistenciales, a lo que debe añadirse los costos generados por pérdida laboral, discapacidad posterior y años de vida prematura perdidos.

Extrapolar los resultados de una institución a lo que ocurre en un país tiene limitaciones. Una de las principales en este caso es que los datos solo representarían lo que ocurre en el subsector privado del SNIS y no en el subsector público. Al comparar las características de la muestra con los de la investigación previa, se observa que el porcentaje de mujeres con IAM fue similar al observado en ambas, pero cuando se discrimina los datos previos, en el subsector público los pacientes fueron más jóvenes que el privado (62,8 vs. 68,4 años de edad promedio) y recibieron menos procedimientos cardiovasculares intervencionistas (60\% vs. 68\%) que en el sector privado 2. Esto, junto a períodos de internación más prolongados, como habitualmente se dan en el subsector público y en las IAMC de Montevideo, podría hacer que los costos asistenciales fuesen diferentes 29. 


\section{Conclusiones}

Las medidas de control de tabaco redujeron los ingresos por IAM, lo que significó una importante mejora en la salud de nuestra población, lo cual se ha visto asociado a un importante ahorro en costos asistenciales para el país. La política de control de tabaco ha sido la principal medida de salud pública implementada en los últimos 30 años en nuestro país.

\section{Colaboradores}

Elbio Paolillo-Cabrera revisó y aprobó la versión final y es responsable de todos los aspectos del trabajo para garantizar la precisión e integridad de cualquier parte del trabajo. F. Torres y E. Sandoya revisaron y aprobaron la versión final. F. Machado, A. Scasso, A. Alvarado, P. Tort, S. Tortorella, S. Laluz y D. Cabrera realizaron el análisis e interpretación de datos y revisaron y aprobaron la versión final. D. Genta escribió el artículo, revisó críticamente el contenido intelectual y aprobó la versión final.

\section{Informaciones adicionales}

ORCID: Elbio Paolillo-Cabrera (0000-0003-45036472); Frank Torres (0000-0002-5774-409X); Federico Machado (0000-0003-2797-3913); Alberto Scasso (0000-0002-3252-7048); Alicia Alvarado (0000-0002-6630-2453); Diego Genta (0000-00018028-8208); Pablo Tort (0000-0002-3091-9718); Silvina Tortorella (0000-0002-6182-5175); Sandra Laluz (0000-0002-9961-2183); Daniela Cabrera (0000-0002-0222-2294); Edgardo Sandoya (00000003-4125-3293).

\section{Agradecimientos}

Queremos manifestar nuestro agradecimiento a Asistencial Médica Departamental de Maldonado (AMDM-IAMPP); Facultad de Medicina del Centro Latinoamericano de Economía Humana (CLAEH); Fondo Nacional de Recursos; Comisión Honoraria para la Salud Cardiovascular; al Centro Cardiológico de Sanatorio Americano; al Prof. Dr. Henry Cohen y a Thais A. Forster MSc. por la colaboración prestada para la realización de la presente investigación.

\section{Referencias}

1. Figueiredo VC, Turci SRB, Camacho LAB. Control del tabaco en Brasil: avances y desafíos de una política bien sucedida. Cad Saúde Pública 2017; 33 Suppl 3:e00104917.

2. Centers for Disease Control and Prevention. Smoking-attributable mortality, years of potential life lost, and productivity losses-United States, 2000-2004. MMWR Morb Mortal Wkly Rep 2008; 57:1226-8.

3. Perez CA, Costa e Silva VL, Bialous SA. Analysis of the tobacco industry's interference in the enforcement of health warnings on tobacco products in Brazil. Cad Saúde Pública 2017; 33 Suppl 3:e00120715.

4. Sandoya E, Bianco E. Mortalidad por tabaquismo y por humo de segunda mano en Uruguay. Rev Urug Cardiol 2011; 26:201-6.

5. Ministerio de Salud Pública. El hábito del tabaco aún causa unas 6.500 muertes por año en Uruguay. https://www.presidencia.gub.uy/ comunicacion/comunicacionnoticias/estudiomsp-enfermedades-atribuibles-tabaco (accedido el 22/Ago/2019).

6. González Mora F, Barbero Portela M. El control del tabaco en Uruguay en perspectiva histórica. Montevideo: Comisión Honoraria para la Salud Cardiovascular/Ministerio de Salud; 2020.

7. Instituto Nacional de Estadística. Censos 2011. http://www.ine.gub.uy/censos-2011 (accedido el 22/Ago/2019).

8. Uruguay. Ley no 18.211 , de 5 de diciembre de 2007. Creación del Sistema Nacional Integrado de Salud. Diario Oficial 2007; 3 dic.

9. Uruguay. Ley no 18.256 , de 6 de marzo de 2008. Control de tabaquismo. Diario Oficial 2008; 10 mar. 
10. Fort Z, Castro M, Portos A, Sandoya E. Evolución del tabaquismo en Uruguay (2006-2011), papel de la enfermera en la promoción de la cesación. Enfermeria (Montev.) 2014; 3:28-32.

11. Hyland A, Travers M, Dresler C, Higbee C, Cummings KM. A 32-country comparison of tobacco smoke derived particle levels in indoor public places. Tob Control 2008; 17:15965.

12. Kalkhoran S, Sebrié EM, Sandoya E, Glantz SA. Effect of Uruguay's national 100\% smokefree law on emergency visits for bronchospasm. Am J Prev Med 2015; 49:85-8.

13. Sebrié EM, Sandoya E, Bianco E, Hyland A, Cummings KM, Glantz SA. Hospital admissions for acute myocardial infarction before and after implementation of a comprehensive smoke-free policy in Uruguay: experience through 2010. Tob Control 2014; 23:471-2.

14. Torres F. Fibrinolíticos en el infarto agudo de miocardio: análisis de una cohorte uruguaya en un período de cuatro años. Rev Urug Cardiol 2017; 32:121-31.

15. Sandoya E, Rivedieu F, Machado F, Susquilvide J, Torres F. Situación actual de la hipertensión arterial en Maldonado. Rev Urug Cardiol 2015; 30:169-75.

16. Fetter R, Shin Y, Freeman JL, Averrill R, Thompson JD. Case mix definition by diagnosis-related groups. Med Care 1980; $18(2$ Suppl):iii, 1-53.

17. Paolillo E, Russi U, Cabrera D, Martins L, Scasso A, Constantin M, et al. Grupos Relacionados por el Diagnóstico (GRD). Experiencia con IR-GRD en Sanatorio Americano, sistema FEMI. Rev Med Urug 2008; 24:257-65.

18. Paolillo E, Scasso A, Torres F, Barrios G, Tavares $\mathrm{G}, \mathrm{Ahmed} Z$, et al. Siniestros de tránsito, los Grupos Relacionados por el Diagnóstico y los costos hospitalarios. Características clínicas y costos de 740 pacientes hospitalizados por siniestros de tránsito en el trienio 2012-2014 en la Asistencial Médica Departamental de Maldonado, Uruguay. Rev Med Urug 2016; 32:2535.

19. Noronha MF, Portela MC, Lebrão ML. Potenciais usos dos AP-DRG para discriminar o perfil da assistência de unidades hospitalares. Cad Saúde Pública 2004; 20 Suppl 2:S242-55.

20. Gorbanev I, Cortés A, Agudelo S, Yepes FJ. ¿Por qué los grupos relacionados de diagnóstico no se implementan en Colombia? Cad Saúde Pública 2015; 31:2027-31.
21. Cortés A, Gorbanev I, Agudelo S, Yepes F. Grupos relacionados por el diagnóstico: experiencia en tres hospitales de alta complejidad en Colombia. Univ Med 2016; 57:171-81.

22. Thygesen K, Alpert JS, White HD; Joint ESC/ ACCF/AHA/WHF Task Force for the Redefinition of Myocardial Infarction. Universal definition of myocardial infarction. Eur Heart J 2007; 28:2525-38.

23. Sexto Grupo de Trabajo Conjunto de la Sociedad Europea de Cardiología y otras Sociedades sobre Prevención de Enfermedad Cardiovascular en la Práctica Clínica. Guía ESC 2016 sobre prevención de la enfermedad cardiovascular en la práctica clínica. Rev Esp Cardiol 2016; 69:939.e1-939.e87.

24. Amrizal MN, Rohaizat Y, Zafar A, Saperi, Syed A. Case-mix costing in Universiti Kebangsaan Malaysia hospital. A top-down approach: cost analysis for cardiology cases. Malaysian Journal of Public Health Medicine 2005; 5 Suppl 2:33-44.

25. Ali Jadoo SA, Aljunid SM, Amrizal MN, Zafar A, Dort DV. Development of MY-DRG casemix pharmacy service weight in UKM Medical Centre in Malaysia. Daru 2015; 23:14.

26. Zafirah SA, Muhammad A, Wan S, Aljunid S. Potential loss of revenue due to errors in clinical coding during the implementation of the Malaysia diagnosis related group (MY-DRG) casemix system in a teaching hospital in Malaysia. BMC Health Serv Res 2018; 18:38.

27. Organización Panamericana de la Salud. Clasificación internacional de enfermedades y problemas relacionados con la salud. 10a revisión. Washington DC: Organización Panamericana de la Salud; 1995. (Publicación Científica, 554).

28. Ministerio de Sanidad, Servicios Sociales e Igualdad. Clasificación Internacional de Enfermedades. 9a revisión - modificación clínica. Madrid: Ministerio de Sanidad, Servicios Sociales e Igualdad; 2014. (Informes y Estadísticas Sanitarias 2013).

29. Instituto Nacional de Estadística. Anuario estadístico 2016. Montevideo: Instituto Nacional de Estadística; 2017. 


\section{Abstract}

Tobacco is the leading modifiable cause of cardiovascular disease, cancer, and respiratory diseases and is thus a serious global public health problem. In 2006, Uruguay implemented the World Health Organization Framework Convention on Tobacco Control (WHO-FCTC) and achieved a decrease in the smoking rate and improvements in cardiovascular and respiratory health. We analyzed the clinical and economic impacts of tobacco control measures on the healthcare costs for acute myocardial infarction, which was reduced by $17 \%$. The costs avoided for other diseases were not included. The study examined the trend in a healthcare institution and projected the result to the country's population. The cost analysis used the diagnosis-related groups (DRG) methodology, combined with the institution's accounting reports. Besides the hospitalization costs, the analysis included patient transportation, invasive cardiovascular procedures, and healthcare costs for the 12 months following the acute myocardial infarction. The cost per patient was USD 12,037. Considering a decrease of 500 acute myocardial infarctions per year, the estimated annual savings are USD 6 million in medical care costs for the averted acute myocardial infarctions, besides savings from averted work absenteeism, subsequent disability, and disability adjusted life years. This successful tobacco control policy has been the leading public health intervention in the last 30 years in Uruguay. The study aims to contribute to the guidelines determined by the World Health Organization (WHO).

Tobacco; Costs and Cost Analysis; Myocardial Infarction

\section{Resumo}

O tabaco é a principal causa modificável de doença cardiovascular, câncer e doenças respiratórias, o que faz dele um sério problema de saúde pública universal. Em 2006, Uruguai implementou a Convenção-Quadro para o Controle do Tabaco da Organização Mundial da Saúde (CQCT-OMS), registrando desde então uma queda na taxa de fumantes, além de melhoras na saúde cardiovascular e respiratória. Foi avaliado o impacto económico e clínico das medidas de controle do tabaco sobre os custos da atenção ao infarto agudo do miocárdio, que diminuíram 17\%. Não foram computados os custos evitados de outras patologias. A pesquisa investigou os registros em uma unidade de saúde e operou uma projeção para toda a população do país. A análise de custos foi desenvolvida usando a metodologia do sistema de Grupos Relacionados pelo Diagnóstico (GRD), combinada com os relatórios contábeis da instituição. Além dos custos de internação, foram incluídos aqueles relativos aos traslados, aos procedimentos cardiovasculares invasivos e às despesas em saúde dos 12 meses posteriores ao evento agudo. O custo por paciente foi de USD 12.037. Considerando uma diminuição de 500 infartos por ano, a economia anual estimada representa USD 6.000.000 na atenção aos infartos evitados; devem ser acrescentadas outras economias de custos de licença médica, invalidez posterior e anos de vida prematura perdidos por óbito do paciente. A bem-sucedida política de controle de tabaco foi a principal medida de saúde pública nos últimos 30 anos, no nosso país. Esta pesquisa pretende contribuir com o caminho trilhado pela Organização Mundial da Saúde (OMS).

Tabaco; Custos e Análise de Custo; Infarto Agudo do Miocárdio
Recibido el 04/Sep/2019

Versión final presentada el 13/May/2020

Aprobado el 20/May/2020 